The following pages constitute the final, accepted and revised manuscript of the article:

Obrant KJ, Ivaska KK, Gerdhem P, Alatalo SL, Pettersson K, Vaananen $\underline{H K}$. "Biochemical markers of bone turnover are influenced by recently sustained fracture."

Bone. 2005 May;36(5):786-92.

Publisher: Elsevier.

Use of alternative location to go to the published version of the article requires journal subscription.

Alternative location:

http://dx.doi.org/10.1016/j.bone.2005.02.009 
From the Departments of Orthopedics*, Malmö University Hospital, SE-205 02 Malmö and the Institute of Biomedicine, Department of Anatomy** and Department of Biotechnology***, University of Turku, Turku, Finland.

\section{Biochemical markers of bone turnover are influenced by} recently sustained fracture.

Karl J.Obrant*, Kaisa K. Ivaska**, Paul Gerdhem*, Sari L. Alatalo**, Kim Pettersson***, H. Kalervo Väänänen**.

Corresponding author: Karl J Obrant, MD, Department of Orthopedics, Malmö University Hospital, 20502 Malmö, Sweden. Fax: +4640336933, e-mail: karl.obrant@orto.mas.lu.se

E-mail addresses to the other authors: paul.gerdhem@skane.se, kaisa.ivaska@utu.fi, sari.alatalo@veripalvelu.fi,kim.pettersson@utu.fi,kalervo.vaananen@utu.fi

Financial support was obtained from the Swedish Research Council*, The Academy of Finland and the National Graduate School for Musculoskeletal Diseases (K.K.I., S.L.A.)**. 


\begin{abstract}
In striving to refine the clinical utility of different markers of bone metabolism, we should take into account numerous confounders, many of which are well known, such as sampling time, fasting status and bone density. One further confounder may be ongoing fracture healing and/or post-fracture immobilisation, which at least theoretically should impose an increased bone formation and resorption. Since both recent fracture and high bone turnover are independent predictors for new fracture, we thought it of importance to define the potential influence of such fracture on markers of bone turnover.
\end{abstract}

From a population-based cohort of 1604 women, all 75 years old (the OPRA-study), 1024 women attended a clinical examination. The bone metabolism was assessed in serum, by three markers of bone formation (bone-specific alkaline phosphatase (S-Bone ALP), intact and NMid osteocalcin (S-Total OC) and total carboxylated osteocalcin (S-cOC)), two markers of bone resorption (C-terminal cross-linked telopeptides of type I collagen (S-CTX) and tartrate resistant acid phosphatase type 5b (S-TRACP5b)), and in urine by one marker of bone resorption (deoxypyridinoline/creatinine (U-DPD/crea)) and two putative markers of bone resorption (urinary osteocalcins (U-OC/crea)). Current physical activity and retrospective fracture data were recorded by questionnaires. The fracture data, for the entire cohort of 1604 women, were validated with radiographic referrals and reports, saved since the beginning of the last century. All data provided, except date of occurrence of retrospectively sustained fracture, were thus obtained cross-sectionally and in all women at the age of 75 .

Fracture had ever been sustained by 727 of the entire cohort $(n=1604)$, and by 523 of the attending women $(n=1024)$. All markers were marginally higher (significant only for U$\mathrm{DPD} / \mathrm{crea}, \mathrm{p}=0.027$ ) in women who had ever sustained fracture, compared to women without 
fracture. In women with recent retrospective fracture (since two years) $(n=100)$, the levels of all markers, except the two S-OCs, were significantly higher $(r=0.20-0.33, p=0.049-0.001)$ the more recently the fracture had been sustained. Women with low current physical activity had elevated levels of U-DPD/crea $(\mathrm{p}<0.001)$ and one U-OC $(\mathrm{p}=0.014)$, while the other markers were unaffected.

Key words: Bone, Marker, Biochemical, Fracture, Physical activity. 


\section{Introduction}

It is reasonable to believe that recently sustained fracture has implications on bone turnover. It has been shown by us and by others [1-4] that a fracture subsequently results in local osteopenia surrounding the fracture site. From histomorphometric analysis of such bone, we know that post-traumatic osteopenia is the result of a high turnover condition, where evidently the bone resorption exceeds that of bone formation [5 6]. Furthermore, the fracture healing events may directly have implications on bone formation, and the relative immobilisation following fracture may contribute to an increased bone resorption.

In spite of the well-grounded body of evidence for the general existence of a post-traumatic skeletal high turnover condition, researchers have, by using biochemical markers of bone turnover, not succeeded to fully demonstrate this condition. One reason for this has been a lack of knowledge about baseline levels, before the fracture event, when fractured patients have been followed longitudinally [7] [8]. Other reasons may have been limited number of individuals investigated, or limited information about fracture history, to draw full conclusions.

Biochemical markers of bone turnover have been used for prediction of fracture, by us and by others [9-19]. If baseline levels of these markers would be influenced in either direction by recently or non-recently sustained fracture, such an event would introduce a possible bias, to be controlled for in further studies on prediction of fracture by biochemical markers. This study was done in order to elucidate whether a retrospectively sustained fracture would have any influence on biochemical markers of bone formation or resorption, and if so, whether such an effect is time dependent. Furthermore, we wanted to find out whether a 
relative immobilisation, taking place after fracture, has any implications on biochemical markers of bone turnover.

\section{Methods}

\section{Subjects}

Invited to the study were 1604 women, all 75 years old. The women were identified at random from the city files of Malmö, Sweden. No exclusion criteria were used. The invitation letter was sent out the week following the $75^{\text {th }}$ birthday of each woman. Of these, 1024 (64\%) women participated in a clinical investigation, where blood and urine samples were collected for assessments of biochemical markers of bone turnover. Reasons for not participating have been reported earlier [20] and were mainly due to unwillingness ( $n=376)$, illness $(n=139)$, failed contact $(n=32)$ or death $(n=13)$ before the day of examination. The study was in all parts approved by the local ethics committee.

\section{Fractures}

All retrospectively sustained fractures from birth to age 75 were ascertained by three means. Firstly, the files of the department of radiology at the Malmö University Hospital were searched for all fractures in the 1604 invited women. This is the only hospital serving the city (260 000 inhabitants), and there is, and always has been, only one department of radiology and one department of orthopaedics, which is the only department where fractures are treated in the city. Due to the personal identification number of each Swedish citizen, retrieval of such data is possible, as is avoidance of double entries. This system also allows us to ensure fracture data when a citizen of Malmö has been treated for fracture at another hospital. Secondly, participating women responded to a questionnaire about retrospectively sustained 
fractures. Thirdly, this information was confirmed against the files of the departments of radiology and orthopaedics.

\section{Sampling procedures}

Non-fasting blood samples were obtained between 08.00 and 13.00. The blood samples were centrifuged and processed within 2 hours after phlebotomy, and stored at $-80^{\circ} \mathrm{C}$. The urine samples were obtained as first morning void and stored at $-80^{\circ} \mathrm{C}$.

\section{Bone markers - formation}

All samples were analysed at the same time. Inter- and intra-assay coefficients of variation (CV) for the assays have been determined previously at our laboratories. Serum bone-specific alkaline phosphatase (S-Bone ALP) was determined using the Metra BAP immunoassay (Quidel Corporation, San Diego, CA, USA), with inter- and intra-assay CV of $4.4 \%$ and 3.6\%, respectively. Serum intact and N-Mid osteocalcin were determined using the Elecsys N-MID Osteocalcin immunoassay (S-Total OC; N-MID®; Roche Diagnostics, Mannheim, Germany), with inter- and intra-assay CV of $2.4 \%$ and $2.3 \%$, respectively. Serum total carboxylated osteocalcin (S-cOC) was determined by a previously published, in-house assay ${ }^{[21]}$, with intra- and inter-assay CV of less than $5 \%$ and $8 \%$.

\section{Bone markers- resorption}

Serum C-terminal cross-linked telopeptides of type I collagen (S-CTX) were determined using the Elecsys $®$-CrossLaps immunoassay (Roche Diagnostics)(intra-assay CV 5.9\%, interassay CV 5.8\%), serum tartrate resistant acid phosphatase type 5b (S-TRACP5b) by a solidphase, immunofixed, enzyme activity assay as described earlier (intra-assay CV 1.8\%, 
interassay CV 2.2\%) ${ }^{[22]}$ and urinary deoxypyridinoline/creatinine (U-DPD/crea) by the Metra DPD immunoassay (intra-assay CV of less than 12\%, inter-assay CV of less than $10 \%$ ) (Quidel Corporation, San Diego, CA, USA).

\section{Bone markers- urine osteocalcin}

Urine osteocalcin (U-OC) was analysed using two novel, two-site assays, U-MidOC/crea and U-LongOC/crea modified from previously described protocols for serum human osteocalcin [21]. The assay U-MidOC/crea measures all the predominant urine OC fragments, detectable by a two-site immunoassay. In contrast, the assay U-LongOC/crea detects only the longest urine OC fragments [23]. The intra-assay CV's are less than 5\%, and interassay CV's less than $8 \%$ for both assays [23].

\section{Other measurements}

Urinary creatinine was determined in accordance with the kinetic Jaffé reaction with a Beckman synchron LX20-4, with CV's of 3\% or less. In addition, body weight and time of specimen collection was recorded, as was serum creatinine analysed by standardised methods.

\section{Self-assessed physical activity}

A self-assessed questionnaire (earlier validated [24]) was used to estimate the present activity into eight physical activity levels from 1) bed rest, cannot walk; 2) walking possible with human support only; 3) walking with walking aid, only in-doors; 4) walking with walking aid, in- and out-doors; 5) no walking aid, walks independently without limitation; 6) takes frequent or regular walks; 7) participates in physical activity more strenuous than walking only; and 8) still working, no limitations of mobility. 


\section{Statistics}

The frequency of retrospectively sustained fracture between the whole population $(n=1604)$ and the women who had their blood and urine sampled $(n=1024)$ was compared with Chisquare analysis. The Shapiro-Wilk test for normal distribution was applied for all markers. STRACP5b, but no other marker, was normally distributed (SW-W $\geq 0,95)$. Statistical calculations were therefore done for all markers, except S-TRACP5b, only after logarithmic transformation. Students t-test was applied when comparison was made between women with retrospective fracture and women without such fracture, or when women with retrospective fracture sustained recently (from age 73.0) were compared with all other women. Furthermore, in women with recent fracture, the age at the last fracture was compared with the serum or urinary concentrations of bone markers with Pearsons regression. To illustrate marker levels in relation to age at latest retrospective fracture, on a Robust locally-weighted regression method (Lowess smoothing) was used. Marker levels in relation to current level of physical activity were compared with one-way analysis of variance.

We used Statistica 6.1 from Statsoft Inc., OK, USA, for all statistical calculations.

\section{Results}

\section{Fractures}

Of all 1604 women invited, 727 (45\%) had had at least one retrospectively sustained fracture. The comparing figure for the women who had their blood and urine sampled $(n=1024)$ was 523 (51\%), a non-significant difference. Of the 1024 sampled women 100, had sustained at least one fracture after the age of 73.0.

The total number of fractures sustained by all women was 1225, and by the sampled women, 908. Of the 1225 fractures sustained by all women, 360 were distal radius fractures, 109 
vertebral fractures, 89 ankle fractures, 76 fractures of the surgical neck of the humerus and 75 hip fractures.

All formation and resorption markers were marginally higher (significant, only for U$\mathrm{DPD} / \mathrm{crea}(\mathrm{p}=0.027))$ in women who ever had sustained retrospective fracture, compared to women without such fracture (Table 1). When plotting the marker levels in relation to age at latest fracture, a visual estimation revealed a tendency for all markers to be higher in those women who had had a fracture within a time period of a few years before sampling (Figure1). There was no difference in body weight $(\mathrm{p}=0.41)$, serum sampling time of the day $(\mathrm{p}=0.26)$ or serum creatinine $(\mathrm{p}=0.16)$ between women with or without ever having a retrospective fracture.

When statistical comparisons were done between women who had fractured themselves after age 73.0 with all other women, S-TRACP5b $(p=0.020)$ and U-LongOC/crea $(p=0.040)$ were higher in the fractured women compared to the others (Table 1). Again, when only women with retrospective fracture since age 73.0 were taken into account, the levels of all markers, except the two serum osteocalcins, were significantly higher $(r=0.20-0.33, \mathrm{p}=0.049-0.001)$ in women the more recently the fracture had been sustained (Table 2, Figures 2 and 3).

One of the 1024 sampled women was always confined to bed (physical activity class 1 ). This single woman was therefore excluded from further analysis. Women with ever a retrospective fracture were currently less physically active, than were the never-fractured women $(\mathrm{p}<0.001)$. The resorption marker U-DPD/crea $(\mathrm{p}<0.001)$ and the urinary osteocalcin ULongOC/crea $(\mathrm{p}=0.014)$ were higher in the least active women compared to the most active 
ones (Figures 4a and 4b), while there was no clear activity-dependent pattern for any of the other markers.

\section{Discussion}

We have failed to find any studies in which the specific aim was to demonstrate levels of different markers of bone turnover in relation to earlier sustained fracture. In a few studies dealing partially with this subject, a firm conclusion has not been reached, partly due to insufficient number of individuals followed up [9 25 26]. The present study involved a sufficient number of women with a sufficient number of fractures sustained, also within a few years prior to sampling, to overcome such a power problem.

The most significant finding in this study is that women with recently sustained fracture have high levels of bone markers, in particular, S-TRACP5b and U-LongOC/crea. Furthermore, within two years, also the formation marker, S-Bone ALP, and the resorption markers, S-CTX and U-DPD/crea, and the urinary Osteocalcin, U-MidOC/crea, were higher the more recently before sampling the fracture had been sustained.

The cutoff limit of two years prior to sampling was chosen based on clinical and scintigraphic experience of fracture consolidation. Interestingly, the results were the same, except for SCTX which no longer was time dependent, had the cut-off limit instead been chosen to one year prior to sampling (data not shown). On the other hand, this study shows that having ever sustained a fracture seems to influence marker levels in a limited way. Although the absolute levels were somewhat higher in the ever-fractured women compared to the never-fractured women, a significantly higher level was only found for U-DPD/crea. 
Neither total (S-Total OC) nor the carboxylated form (S-cOC) of osteocalcin seemed to be influenced in any way by a recently sustained fracture, while the third marker of bone formation analysed, S-Bone ALP, was increased. This is in line with earlier findings that osteocalcin seems to be involved in the early phase of mineralization after fracture, while its presence seems not to be a requirement for the continuous mineralization process [27]. Alkaline phosphatase (S-Bone ALP), on the other hand, may be more involved also in the continuous mineralization processes [28]. The different markers may thus reflect different stages of osteoblastic development and function, which would be one possible explanation for the divergent findings of the markers of bone formation. These findings do not exclude, however, that there may be a transient increase in serum osteocalcin after fracture [8].

It is obvious, from a theoretical point of view, that not only bone formation, but also bone resorption, should be increased compared to baseline after fracture. Bone resorption was evaluated in this paper by three generally accepted biochemical markers of resorption (SCTX, S-TRACP5b and U-DPD/crea), and by two markers for which we have some evidence to believe that they are also markers of resorption rather than formation (U-LongOC/crea and U-MidOC/crea) [8] , [19]. All five of these markers were elevated in women with recently sustained fracture. However, only two of them, in particular U-DPD/crea but also ULongOC/crea, were also elevated in those women who were least physically active at the time of sampling. The reason for this latter finding is probably due to the limited discriminative power of the activity scores used. Also, there were considerably less women in the low activity grades compared to the intermediate and high grades, and we cannot exclude that the absence of a connection, for also the other resorption markers, between low physical activity and bone resorption is due to insufficient power of the study for this specific question. It is likely that these five markers actually represent different aspects of bone resorption, whether 
the resorption is directly dependent on the immobilized status or is more of a consequence of the metabolic remodelling events taking place at the specific skeletal fracture location.

There are some specific advantages with the study design used in this paper. All women had the same age, 75, making age corrections unnecessary. In addition all women were of Caucasian ancestry and recruited from the same catchment area, Malmö city, Sweden. Fracture registration throughout life was made possible by the quite unique situation at the department of radiology, in this city, where all radiographs, referrals and reports have been saved for one hundred years. This situation throughout the $20^{\text {th }}$ century has resulted in a considerable number of papers on fracture epidemiology [29]. We cannot exclude, however, that some of the women included in this study, and in particular a long time ago, refrained from radiographic examination after fracture trauma. Therefore, we may have missed some fractures from registration and, in particular, fractures sustained when the women were still young. If so, this would, however, have no influence on the conclusions drawn from this study. Furthermore, the lifetime prevalence of fracture did not differ between participating women (n=1024) and the entire cohort of invited women $(n=1604)$ for whom we were able to search the files and/or register fractures by questioning. This leads us also to believe that the sampled women could be judged to represent fairly well the entire population.

There are also some limitations with this study. Since all the women were of the same age, we cannot generalize the results to be valid for both sexes and at all ages. We find no reason to believe, however, that the results should not be commonly valid for elderly women well above menopausal age. 
For some of the markers, in particular, S-CTX, there is an influence of non fasting status on the serum concentration [30]. At the time of initiating this study in 1995, knowledge of this was not available. In spite of this, S-CTX did appear to react to fracture in a comparable way with the other resorption markers, in particular, S-TRACP5b, which is known to be considerably less dependent of fasting status [31]. In general there was no difference in fasting status between ever-fractured women, and never-fractured women.

There is evidence that retrospectively sustained fracture is a strong independent predictor of new fractures [32 33]. Also, biochemical markers of bone turnover have been used to predict forthcoming fractures [9-17]. In particular, high levels of resorption markers, such as S-CTX [12] and S-TRACP5b [19], have been associated with such fractures. There seems also to be convincing evidence that combining marker levels with bone mineral density measurements would further enhance our possibilities to predict fractures[19] [33]. Ideally, we would learn to use already existing and novel biochemical markers that to some extent can add to, or even replace, today's equipment and personnel required for bone density testing. In order to gain such marker-specific knowledge, we need to characterize all the different factors that may influence one or another marker. In conclusion, the results from assessments of markers of bone turnover should be used, with caution if the patient has sustained a fracture within two years prior to sampling. 


\section{References}

1. Finsen V, Benum P. Osteopenia after ankle fractures. The influence of early weight bearing and muscle activity. Clin Orthop 1989(245):261-8.

2. Findlay SC, Eastell R, Ingle BM. Measurement of bone adjacent to tibial shaft fracture. Osteoporos Int 2002;13(12):980-9.

3. Andersson SM, Nilsson BE. Post-traumatic bone mineral loss in tibial shaft fractures treated with a weight-bearing brace. Acta Orthop Scand 1979;50(6 Pt 1):689-91.

4. Karlsson MK, Josefsson PO, Nordkvist A, Akesson K, Seeman E, Obrant KJ. Bone loss following tibial osteotomy: a model for evaluating post-traumatic osteopenia. Osteoporos Int 2000;11(3):261-4.

5. Obrant KJ. Trabecular bone changes in the greater trochanter after fracture of the femoral neck. Acta Orthop Scand 1984;55(1):78-82.

6. Obrant KJ, Nilsson BE. Histomorphologic changes in the tibial epiphysis after diaphyseal fracture. Clin Orthop 1984(185):270-5.

7. Ingle BM, Hay SM, Bottjer HM, Eastell R. Changes in bone mass and bone turnover following ankle fracture. Osteoporos Int 1999;10(5):408-15.

8. Akesson K KS-M, Josefsson PO, Karlsson M, Pettersson K, Obrant KJ. Fracture induced changes in bone turnover - A potential confounder in the use of biochemical markers in osteoporosis. Journal of Bone and Mineral Metabolism, in press 2004.

9. Akesson K, Ljunghall S, Jonsson B, Sernbo I, Johnell O, Gardsell P, Obrant KJ. Assessment of biochemical markers of bone metabolism in relation to the occurrence of fracture: a retrospective and prospective population-based study of women. J Bone Miner Res 1995;10(11):1823-9.

10. van Daele PL, Seibel MJ, Burger H, Hofman A, Grobbee DE, van Leeuwen JP, Birkenhager JC, Pols HA. Case-control analysis of bone resorption markers, disability, and hip fracture risk: the Rotterdam study. Bmj 1996;312(7029):482-3.

11. Garnero P, Hausherr E, Chapuy MC, Marcelli C, Grandjean H, Muller C, Cormier C, Breart G, Meunier PJ, Delmas PD. Markers of bone resorption predict hip fracture in elderly women: the EPIDOS Prospective Study. J Bone Miner Res 1996;11(10):15318.

12. Garnero P, Sornay-Rendu E, Claustrat B, Delmas PD. Biochemical markers of bone turnover, endogenous hormones and the risk of fractures in postmenopausal women: the OFELY study. J Bone Miner Res 2000;15(8):1526-36.

13. Garnero P, Cloos P, Sornay-Rendu E, Qvist P, Delmas PD. Type I collagen racemization and isomerization and the risk of fracture in postmenopausal women: the OFELY prospective study. J Bone Miner Res 2002;17(5):826-33.

14. Luukinen H, Kakonen SM, Pettersson K, Koski K, Laippala P, Lovgren T, Kivela SL, Vaananen HK. Strong prediction of fractures among older adults by the ratio of carboxylated to total serum osteocalcin. J Bone Miner Res 2000;15(12):2473-8.

15. Chapurlat RD, Garnero P, Breart G, Meunier PJ, Delmas PD. Serum type I collagen breakdown product (serum CTX) predicts hip fracture risk in elderly women: the EPIDOS study. Bone 2000;27(2):283-6.

16. Bruyere O, Collette J, Delmas P, Rouillon A, Roux C, Seidel L, Richy F, Reginster JY. Interest of biochemical markers of bone turnover for long-term prediction of new vertebral fracture in postmenopausal osteoporotic women. Maturitas 2003;44(4):25965.

17. Ross PD, Kress BC, Parson RE, Wasnich RD, Armour KA, Mizrahi IA. Serum bone alkaline phosphatase and calcaneus bone density predict fractures: a prospective study. Osteoporos Int 2000;11(1):76-82. 
18. Vergnaud P, Garnero P, Meunier PJ, Breart G, Kamihagi K, Delmas PD. Undercarboxylated osteocalcin measured with a specific immunoassay predicts hip fracture in elderly women: the EPIDOS Study. J Clin Endocrinol Metab 1997;82(3):719-24.

19. Gerdhem Paul KKI, Sari L Alatalo, Jussi M Halleen, Jukka Hellman, Anders Isaksson, Kim Pettersson, H. Kalervo Vaananen, Kristina Akesson, Karl J Obrant. Biochemical markers of bone metabolism and prediction of fracture in elderly women. In press 2004.

20. Gerdhem. P. Risk factors for falls and fractures in elderly women. Lund University, 2004.

21. Kakonen SM, Hellman J, Karp M, Laaksonen P, Obrant KJ, Vaananen HK, Lovgren T, Pettersson K. Development and evaluation of three immunofluorometric assays that measure different forms of osteocalcin in serum. Clin Chem 2000;46(3):332-7.

22. Halleen JM, Alatalo SL, Suominen H, Cheng S, Janckila AJ, Vaananen HK. Tartrateresistant acid phosphatase 5b: a novel serum marker of bone resorption. J Bone Miner Res 2000;15(7):1337-45.

23. Ivaska KK, Hellman J, Likojarvi J, Kakonen SM, Gerdhem P, Akesson K, Obrant KJ, Pettersson K, Vaananen HK. Identification of novel proteolytic forms of osteocalcin in human urine. Biochem Biophys Res Commun 2003;306(4):973-80.

24. Gerdhem P, Ringsberg KA, Akesson K, Obrant KJ. Influence of muscle strength, physical activity and weight on bone mass in a population-based sample of 1004 elderly women. Osteoporos Int 2003;14(9):768-72.

25. Melton LJ, 3rd, Khosla S, Atkinson EJ, O'Fallon WM, Riggs BL. Relationship of bone turnover to bone density and fractures. J Bone Miner Res 1997;12(7):1083-91.

26. Lo Cascio V, Bertoldo F, Gambaro G, Gasperi E, Furlan F, Colapietro F, Lo Cascio C, Campagnola M. Urinary galactosyl-hydroxylysine in postmenopausal osteoporotic women: A potential marker of bone fragility. J Bone Miner Res 1999;14(8):1420-4.

27. Lian BJ SG, Gerstenfeld L, Glowacki J. Gene expression and functional studies of the vitamin K-dependent protein of bone, osteocalcin. In: Lindh E, ed. Clinical impact of bone and connective tissue markers. London: Academic Press 1989:121-132.

28. Yu-Yahiro JA, Michael RH, Dubin NH, Fox KM, Sachs M, Hawkes WG, Hebel JR, Zimmerman SI, Shapiro J, Magaziner J. Serum and urine markers of bone metabolism during the year after hip fracture. J Am Geriatr Soc 2001;49(7):877-83.

29. Obrant KJ, Bengner U, Johnell O, Nilsson BE, Sernbo I. Increasing age-adjusted risk of fragility fractures: a sign of increasing osteoporosis in successive generations? Calcif Tissue Int 1989;44(3):157-67.

30. Clowes JA, Hannon RA, Yap TS, Hoyle NR, Blumsohn A, Eastell R. Effect of feeding on bone turnover markers and its impact on biological variability of measurements. Bone 2002;30(6):886-90.

31. Hannon RA, Clowes JA, Eagleton AC, Al Hadari A, Eastell R, Blumsohn A. Clinical performance of immunoreactive tartrate-resistant acid phosphatase isoform $5 \mathrm{~b}$ as a marker of bone resorption. Bone 2004;34(1):187-94.

32. Karlsson MK, Hasserius R, Obrant KJ. The ankle fracture as an index of future fracture risk. A 25-40 year follow-up of 1063 cases. Acta Orthop Scand 1993;64(4):482-4.

33. Kanis JA. Diagnosis of osteoporosis and assessment of fracture risk. Lancet 2002;359(9321):1929-36. 
Table 1. Markers of bone turnover, in women with or without retrospectively sustained fracture. Data are presented as mean \pm standard deviation as assessed at age 75 . Due to nonnormal distributions, statistical comparisons are, except for S-TRACP5b, based on logarithmically transformed data.

\begin{tabular}{|c|c|c|c|c|}
\hline & $\begin{array}{l}\text { Women } \\
\text { with } \\
\text { retrospective } \\
\text { fracture } \\
\text { (n=523) }\end{array}$ & $\begin{array}{l}\text { Women } \\
\text { without } \\
\text { retrospective } \\
\text { fracture } \\
(n=501)\end{array}$ & $\begin{array}{l}\text { Women with } \\
\text { retrospective } \\
\text { fracture } \\
\text { from age } 73.0 \\
(n=100)\end{array}$ & $\begin{array}{l}\text { Women without } \\
\text { retrospective } \\
\text { fracture } \\
\text { from age } 73.0 \\
(n=924)\end{array}$ \\
\hline \multicolumn{5}{|l|}{ Formation markers } \\
\hline S-Bone ALP (U/L) & $22.8+8.8$ & $22.5+8.4$ & $23.4+9.5$ & $22.6+8.5$ \\
\hline S-Total OC (microg/L) & $28.8 \pm 13.0$ & $28.5 \pm 12.7$ & $28.5 \pm 11.4$ & $28.7+13.0$ \\
\hline S-cOC (microg/L) & $7.53 \pm 3.38$ & $7.44 \pm 3.66$ & $7.40 \pm 2.88$ & $7.50 \pm 3.57$ \\
\hline \multicolumn{5}{|l|}{ Resorption markers } \\
\hline S-CTX (ng/L) & $313+213$ & 299+189 & $352+234$ & $302+198$ \\
\hline S-TRACP5b (U/L) & $3.4 \overline{5}+1.20$ & $3.40+1.16$ & $3.69+1.39^{b}$ & $3.40+1.16$ \\
\hline U-DPD/crea (nmol/mmol) & $8.72 \pm 4.44^{\mathrm{a}}$ & $8.16 \pm 4.61$ & $9.26 \pm 5.31$ & $8.36+4.43$ \\
\hline \multicolumn{5}{|l|}{ Urine osteocalcin } \\
\hline U-MidOC/crea (microg/mmol) & $1.31 \pm 1.05$ & $1.22 \pm 0.78$ & $1.33 \pm 0.83$ & $1.26 \pm 0.94$ \\
\hline $\begin{array}{l}\text { U-LongOC/crea } \\
\text { (microg/mmol) }\end{array}$ & $0.07 \pm 0.24$ & $0.05 \pm 0.06$ & $0.09 \pm 0.16^{\mathbf{b}}$ & $0.06 \pm 0.18$ \\
\hline
\end{tabular}


Table 2. Correlations (Pearson's r-value) and levels of significances (p-value) for the different markers of bone turnover in relation to age when last fracture was sustained. Only women with fracture sustained from age 73.0 are included in this table. Statistical comparisons are, except for S-TRACP5b, based on logarithmically transformed data.

\begin{tabular}{lll}
\hline & $r$ value & P value \\
\hline Formation markers & & \\
S-Bone ALP (U/L) & 0.31 & 0.003 \\
S-Total OC (microg/L) & 0.16 & $\mathrm{~ns}$ \\
S-cOC (microg/L) & 0.18 & $\mathrm{~ns}$ \\
& & \\
Resorption markers & & 0.049 \\
S-CTX (ng/L) & 0.20 & 0.002 \\
S-TRACP5b (U/L) & 0.31 & 0.001 \\
U-DPD/crea (nmol/mmol) & 0.33 & \\
Urine osteocalcin & & \\
U-MidOC/crea (microg/mmol) & 0.27 & 0.008 \\
U-LongOC/crea (microg/mmol) & 0.28 & 0.006 \\
\hline
\end{tabular}




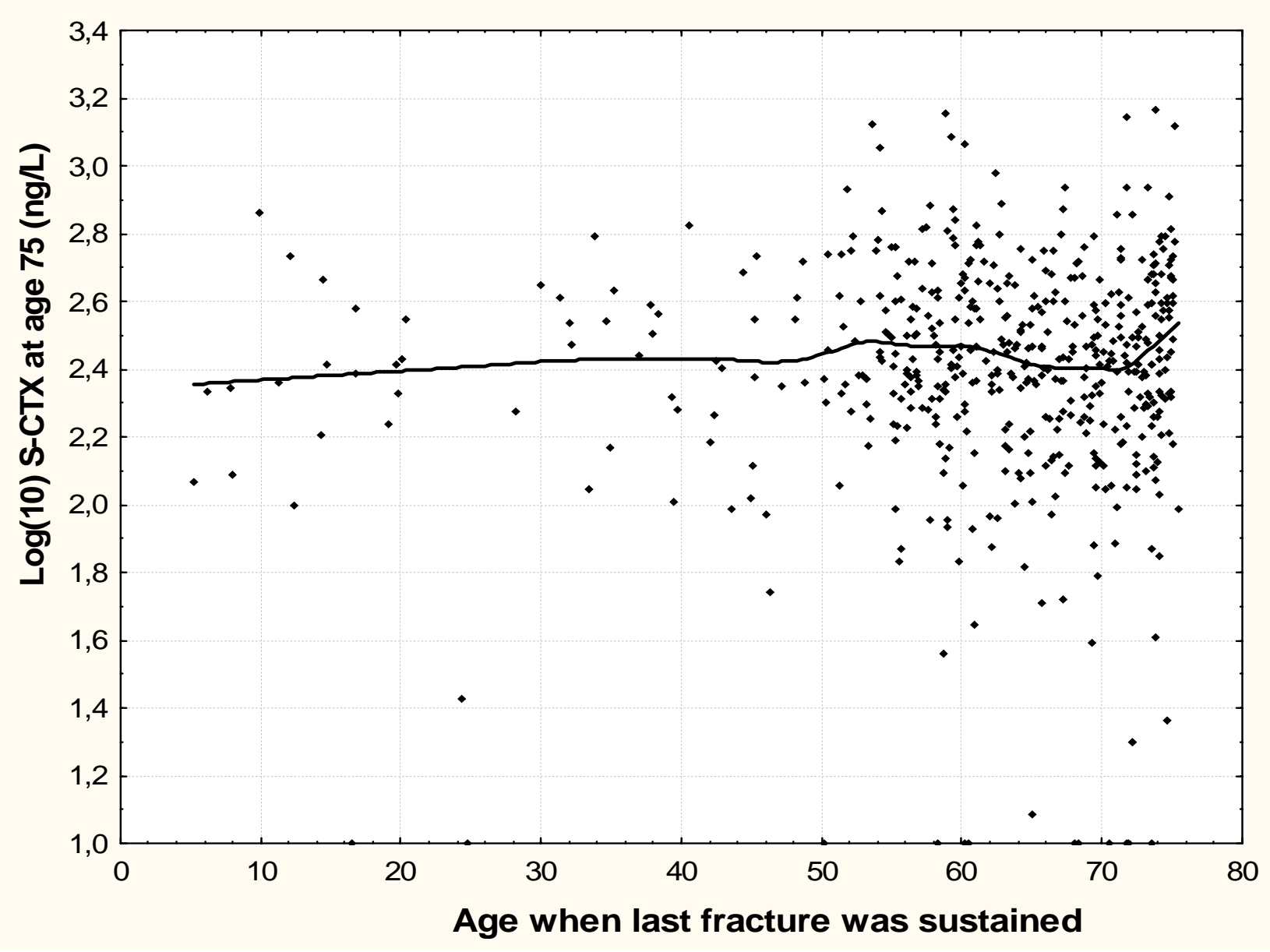

Figure 1.

S-CTX, at age 75, and in relation to age when retrospectively sustained fracture occurred. Women with two or more fractures are denoted only when last fracture occurred. Line indicates a robust locally-weighted regression (Lowess smoothing). 


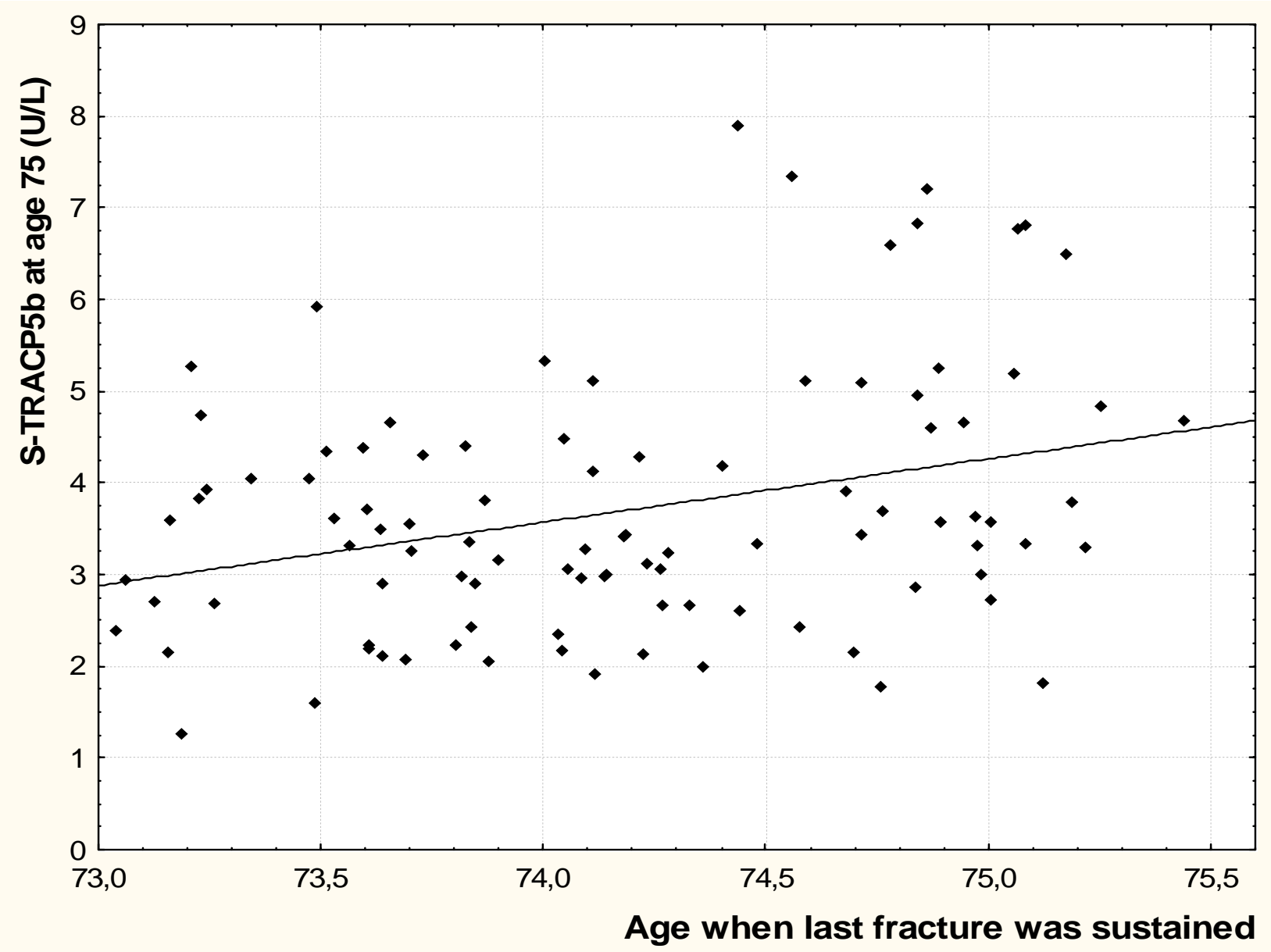

\section{Figure 2.}

S-BALP, at age 75 , and in relation to age at last sustained retrospective fracture. Line indicates Pearson's regression equation. Only women with retrospectively sustained fracture after age 73.0 are included in this figure. Note that the exact age at sampling was $75.23+$ 0.14, meaning that a few of the women had had their last fracture after age 75.0, but always before sampling time. 


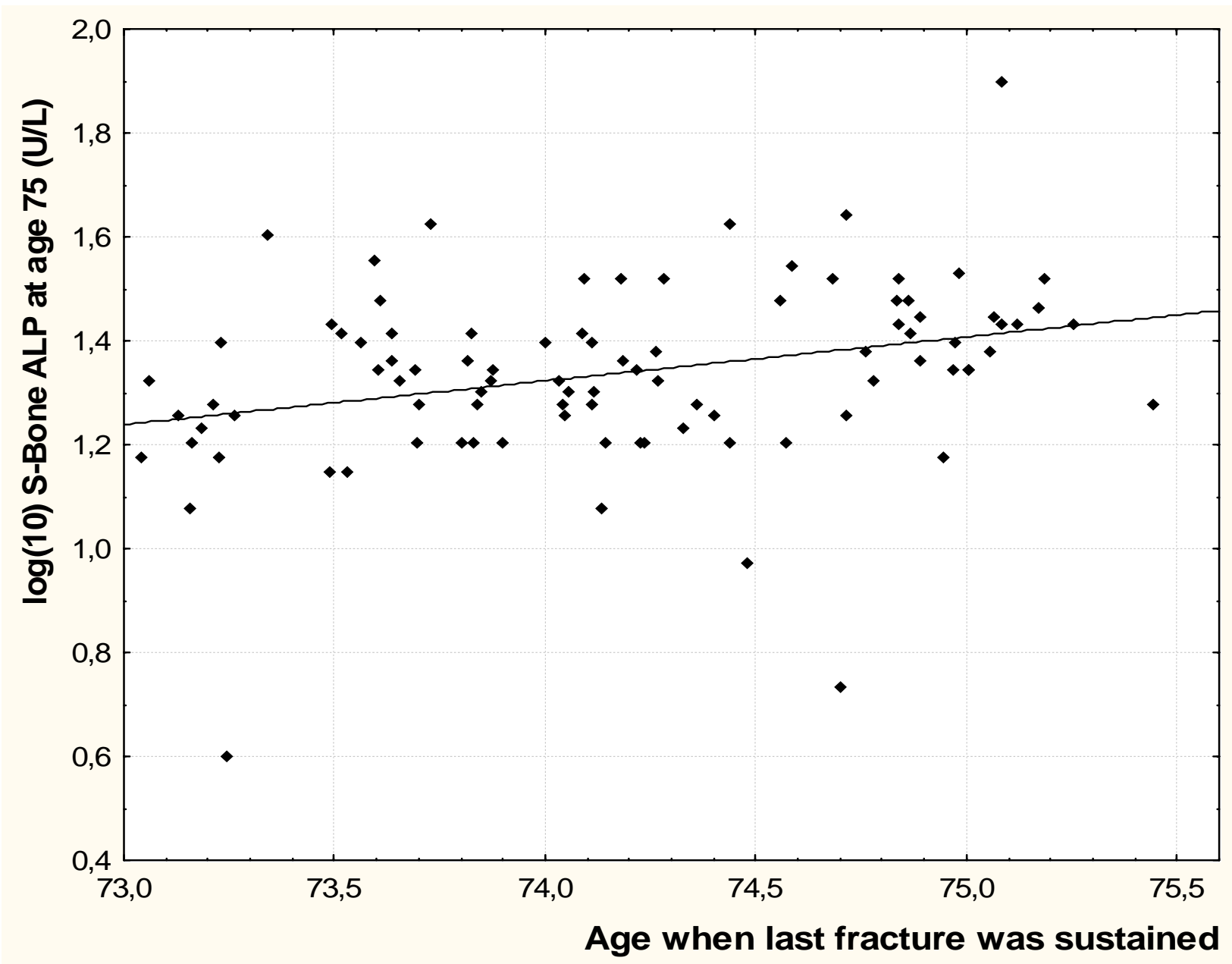

\section{Figure 3.}

S-TRACP5b, at age 75, and in relation to age at last sustained retrospective fracture. Line indicates Pearson's regression equation. Only women with retrospectively sustained fracture after age 73.0 are included in this figure. Note that the exact age at sampling was $75.23 \pm$ 0.14, meaning that a few of the women had had their last fracture after age 75.0, but always before sampling time. 

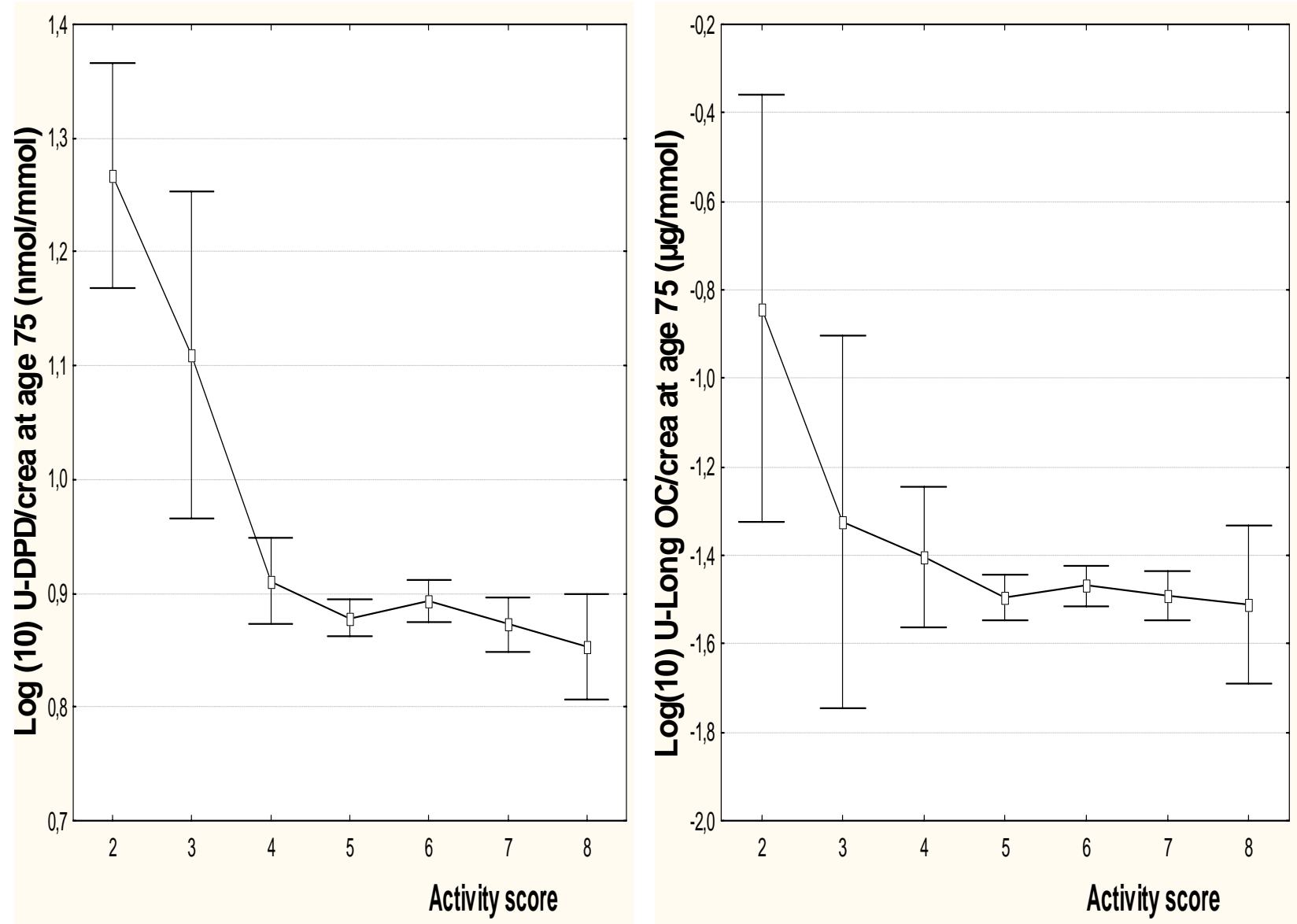

Figures $4 a$ and $b$.

U-DPD/crea and U-LongOC/crea at age 75, in relation to current physical activity (2=low, 8=high, for further information see text)(Mean \pm 0.95 Conf. Interval). 